cardiac murmur. Peripheral pulse quality should be assessed and any pulse deficits should be noted. Mucous membrane colour and capillary refill time should be assessed to evaluate the patient's perfusion status.

\section{ASSESSMENT OF THE RESPIRATORY SYSTEM}

Before handling the emergency patient, visual observations of the patient's chest wall will provide information including respiratory rate, and it can be determined whether the patient has an increased respiratory effort. If the patient is suffering from respiratory distress then oxygen therapy should be administered whilst the patient undergoes any further examination. Thoracic auscultation should be performed to assess for the presence of any abnormal lung sounds.

\section{ASSESSMENT OF THE NEUROLOGICAL SYSTEM}

The neurological assessment of an emergency patient involves assessing the patient's demeanour, whether the patient is able to ambulate and whether the patient has any cranial nerve deficits.

\section{KEY LEARNING OBJECTIVES}

- Understand when it is appropriate to perform a major body system assessment (MBSA)
Understand why we perform MBSAs

Understand how to perform an MBSA

\section{MULTIPLE CHOICE QUESTIONS}

1. During an MBSA of a feline patient, you note that the patient's heart rate is $100 \mathrm{bpm}$. Bradycardia (heart rate less than $120 \mathrm{bpm}$ ) in a cat is most likely to be associated with which of the following conditions?

(A) Allergic bronchitis (feline asthma)

(B) Aortic thromboembolism

(C) Hypovolaemic shock

(D) Severe pain

2. What lung sounds may be noted during thoracic auscultation for a patient that has a pneumothorax?

(A) Increased lung sounds

(B) Increased heart sounds

(C) Decreased or absent lung sounds

(D) Crackles

3. A patient is presented with a suspected gastric dilatation-volvulus. Which of the following is least likely to be a problem?
(A) Hypotension
(B) Cardiac arrhythmias
(C) Cerebral oedema
(D) Respiratory acidosis

\section{Pleural space disease: what are you doing there?}

\section{Jessica Herley}

The pleural space is defined as the area between the lungs and the chest wall. Pleural space disease occurs when air, fluid or tissue occupies this space. Examples of pleural space disease include pneumothorax (air in the pleural space) and pleural effusion (fluid in the pleural space). Pleural space disease may be unilateral or bilateral, depending upon the cause.

Clinical signs of pleural space disease include increased respiratory rate and effort, coughing, fever, weight loss and lethargy. Clinical examination of patients in respiratory distress should include thoracic auscultation during which dull lung sounds are most commonly noted if the patient is suffering from pleural space disease. A pleural effusion can occur secondary to heart disease so cardiac auscultation should also be performed to assess for evidence of a murmur or cardiac arrhythmia.
Pleural space disease can be diagnosed by taking radiographs of the thorax. Alternatively, or additionally, a TFAST (thoracic focused assessment with sonography for trauma) scan may be undertaken to check for the presence of air, tissue or fluid within the pleural space. Thoracocentesis is an invasive procedure that is performed to remove fluid or air from the pleural space. Thoracocentesis should be performed for any patient that has a build up of air or fluid in the pleural space which is affecting their respiratory function. Thoracentesis is a procedure which involves inserting a needle or catheter into the pleural space and withdrawing the air or fluid which has built up within the pleural space. The procedure usually provides immediate relief for the patient and if there is fluid present within the pleural space, this can be collected and sent for laboratory analysis. Patients in respiratory distress may object to the restraint that is required for thoracocentesis. Any distress suffered by a dyspnoeic animal can be life threatening so sedation or anaesthesia may be required to perform thoracocentesis safely. Coagulopathies are a contraindication of thoracocentesis, but sometimes the procedure cannot be avoided, especially in the case of life-threatening pleural haemorrhage.

Treatment of pleural space disease often includes oxygen therapy and the patient will require strict rest. 


\section{KEY LEARNING OBJECTIVES}

- Understand what pleural space disease is

- Understand why pleural space disease occurs

- Understand how to manage patients suffering from pleural space disease

\section{MULTIPLE CHOICE QUESTIONS}

1. A patient is presented with dyspnoea and pleural space disease is suspected to be the cause. What treatment should take priority?
(A) Administration of analgesia

(B) Thoracocentesis
(C) Administration of antibiotic therapy

(D) Oxygen therapy

2. In preparation for a thoracocentesis procedure, which landmark should be clipped and aseptically prepared?

(A) 3 rd-5th rib spaces

(B) 4th-5th rib spaces

(C) 5 th -7 th rib spaces

(D) 7th-9th rib spaces

3. Which of the following conditions is most likely to cause a spontaneous pneumothorax?

(A) Blunt trauma

(B) Penetrating trauma

(C) Bacterial infection

(D) Bullous emphysema
Heat exchange: loss, gain and management demystified

\section{Jessica Herley}

Dogs and cats are endotherms, which means they mostly use metabolic heat to maintain a stable internal temperature, which often is different from the environmental temperature. The body possesses numerous homeostatic abilities in order to maintain an equilibrium and this is referred to as thermoregulation. Thermoregulation is a balance between heat production and heat loss. There are four principle mechanisms for heat loss or heat gain: convection, conduction, radiation and evaporation. Dogs and cats have behavioural, anatomical and physiological adaptation features that help them maintain a normal temperature.

During illness, anaesthesia or when an animal is exposed to extreme environmental temperature changes, thermoregulation may become adversely affected and patients may become hypothermic or hyperthermic.

Hypothermia is characterized by an abnormally low body temperature and can be classified as mild, moderate or severe. Hypothermia most commonly occurs when an animal is exposed to cold environmental temperatures. It can lead to depression of the central nervous system, cardiovascular system, respiratory system and the immune system. Hypothermia symptoms vary with the level of severity. Mild hypothermia can result in weakness, shivering and dull mentation. Moderate hypothermia can result in muscle stiffness, hypotension, stupor state and dyspnoea. Severe hypothermia can result in fixed, dilated pupils, dyspnoea, inaudible heart rate and coma.

Hyperthermia is defined as an elevation in temperature above the normal range for the species. Healthy patients have a 'set-point temperature', which may vary with changing environments or differing activity levels, meaning that homeostatic mechanisms are employed to maintain a temperature within a normal range rather than a fixed value. The thermoregulatory centre, which is located in the hypothalamus, senses any changes from the set-point temperature and sends signals to initiate heat dissipation measures when hyperthermia is detected. Clinical signs of hyperthermia include excessive panting, collapse, ataxia, hypersalivation, vomiting, seizures, diarrhoea, dull mention, haematuria, epistaxis and swollen tongue.

Treatment of hyperthermic or hypothermic patients should be focused on the primary disease process contributing to the derangement.

\section{KEY LEARNING OBJECTIVES}

- Understand how our patients lose and gain heat

- Understand how to manage patients suffering from hypothermia or hyperthermia

- Understand the effects that hypothermia and hyperthermia have on the body

\section{MULTIPLE CHOICE QUESTIONS}

1. Which of the following is another term for fever?
(A) Hypothermia
(C) Pyrexia
(B) Hyperthermia
(D) Isothermia

2. Where are the peripheral thermoreceptors found?

(A) Skin and mucous membranes

(B) Spinal cord

(C) Liver

(D) Brain

3. Which mechanism of heat dissipation is characterized by heat being transferred from the patient to a cooler object?
(A) Convection
(C) Conduction
(B) Radiation
(D) Evaporation 